\title{
Mupirocin Resistance in Coagulase Negative Staphylococcus Isolated from Rural Population - An Underestimated Threat?
}

\author{
Dinesh Kumar' ${ }^{1}$, Dakshina Bisht², Sameer Singh Faujdar ${ }^{3}$ \\ 1,2 Department of Microbiology, Santosh Medical College \& Hospital, \\ Ghaziabad Uttar Pradesh, India. ${ }^{3}$ Department of Microbiology, Maharishi \\ Markandeshwar Medical College \& Hospital, Solan, Himachal Pradesh, India.
}

\section{ABSTRACT}

\section{BACKGROUND}

CoNS when exposed to repeated or prolonged treatment with mupirocin ointment, may become a reservoir of high-level resistance determinants and then pass on this resistance to $S$. aureus. CoNS usually tends to be reservoirs of antimicrobial resistance factors, hence they generally lead to recurrence of multi-drug resistance. Hence, it is imperative to identify and discriminate the strains of S. aureus and CoNS. We wanted to analyse mupirocin resistance in coagulase negative Staphylococcus isolated from a rural population.

\section{METHODS}

This study was performed in the Department of Microbiology, SMCH (Ghaziabad) among indoor as well as OPD patients of a tertiary care hospital. All coagulase negative Staphylococcus strains were taken from patient's clinical specimens visiting the OPD or from patients getting treatment from the hospital. CoNS were identified by standard biochemical tests. An inhibition zonal area $<21 \mathrm{~mm}$ was considered as resistant.

\section{RESULTS}

Mupirocin resistance was found to be $7 \%$ in MRCoNS and $5 \%$ in MSCoNS. MupRH and MupRL were $5 \%$ and $7 \%$ respectively. Only 5 (5\%) isolates showed MIC more than $512 \mu \mathrm{g} / \mathrm{mL}$ as described.

\section{CONCLUSIONS}

In case of emergence of mupirocin resistance, other decolonization options ought to be considered.

\section{KEY WORDS}

CoNS, Methicillin-Resistant, High-Level, Low-Level, Mupirocin Resistance
Corresponding Author:

Dr. Dakshina Bisht.

Department of Microbiology, Santosh

Medical College \& Hospital, Ghaziabad

Uttar Pradesh, India.

E-mail: dakshinabisht@gmail.com

DOI: $10.14260 / j e m d s / 2020 / 668$

How to Cite This Article:

Kumar D, Bisht D, Faujdar SS. Mupirocin resistance in coagulase negative staphylococcus isolated from rural population - an underestimated threat? J Evolution Med Dent Sci 2020;9(41):30503054, DOI: $10.14260 /$ jemds/2020/668

Submission 22-06-2020,

Peer Review 06-09-2020,

Acceptance 12-09-2020,

Published 12-10-2020.

Copyright (C) 2020 Dinesh Kumar et al. This is an open access article distributed under Creative Commons Attribution License [Attribution 4.0 International (CC BY 4.0)] 


\section{BACKGROUND}

Coagulase-Negative Staphylococcus (CoNS) until recently was considered as a non-harmful commensal microflora of human body but now is emerging as an important reason of hospital acquired as well as community acquired infections. CoNS is the leading cause of medical implant device-related infections, nosocomial blood-stream infections, and account substantially for infections in the preterm and low-birth-weight newborns. ${ }^{1-}$ 2 They give rise to various clinical infections including infective endocarditis especially when the species and strains are unusually virulent. ${ }^{3}$ The significance of CoNS posing as a greater nosocomial micro-flora in regards to prosthesis and indwelling device-associated infections is identified. CoNS also is the most usually isolated flora in microbiological laboratories. 4 Of importance, CoNS usually tends to be reservoirs of antimicrobial resistance factors, hence they generally present a great recurrence for multi-drug resistance. ${ }^{5}$ Hence, it is imperative to identify and discriminate the strains of $S$. aureus and CoNS.

The incidence of infections caused by this group of organisms is mostly under-reported because of the difficulty in identifying its pathogenic role; even by using molecular techniques, colonization, and infection cannot be differentiated. ${ }^{6}$ Methicillin resistance has contributed to the emergence of CoNS as a common cause of nosocomial infections. Methicillin resistance in CoNS may not only lead to treatment failure but also spread this resistance to Staphylococcus aureus which may pose a challenge to clinicians.7 "These strains show confrontation to penicillins, clindamycin, tetracyclines, macrolides, linezolid, methicillin (MET) and now a days to mupirocin (MUP) and vancomycin. This resistance constraints therapeutic choices for intervention and principally enhances case morbidity and mortality".

Indiscriminate use of mupirocin, a topical antibiotic that is employed for eradication of staphylococcal nasal colonization to prevent healthcare-associated methicillin-resistant staphylococcal infections has already led to the emergence of its resistance. CoNS on nasal mucosal surfaces, when exposed to repeated or prolonged treatment with mupirocin ointment, may become a reservoir of high-level resistance determinants and then pass on this resistance to $S$. aureus. The events of gene transfer are most prone to show up at locations colonized by CoNS and coagulase positive staphylococci such as the normal nasal mucosa. ${ }^{8}$

"The resistance issue prevalent globally make laboratories to establish quick, precise, method for characterizing as well as distinguishing MRSA, MUP-resistant $S$. aureus, and MUPresistant CoNS. PCR is the most common technique used for the same but scarce literature is available". ${ }^{9}$ Hence the current study was done to know about mupirocin resistnace status in CoNS, isolated from rural population of UP.

\section{METHODS}

This is a prospective study was performed in the Department of Microbiology, Santosh Medical College and Hospital, Ghaziabad, with authorization from the Institutional Ethical
Committee (F. No. SU / 2017 / 683 (15); 26 / 05 / 2017) in indoor and outpatient departments.

All $S$. CoNS strains were taken from patients clinical specimens visiting the OPD or getting treatments from the hospital. Samples acquired in the laboratory were taken care for isolation and identification of CoNS isolates. Samples eliminating urine inoculated on 5 percent blood agar and MacConkey agar media, while urine specimens were inoculated on cysteine-lactose electrolyte deficient (CLED) agar media and incubated at $37^{\circ} \mathrm{C}$ aerobically. The growth was recognized as CoNS with the help of standard biochemical methods". 10

\section{Antimicrobial Susceptibility Testing}

"Checking of antimicrobial susceptibility (AST) on all isolates as per the Clinical and Laboratory Standards Institute (CLSI) guidelines isolates were made on Mueller-Hinton agar media (HiMedia) by Kirby-Bauer disc diffusion technique. Isolate inoculum with turbidity of 0.5 McFarland norm (1.5 some 108 $\mathrm{CFU} / \mathrm{ml}$ ) was prepared in peptone water and lawn-culture was prepared on Mueller Hinton agar (MHA) and allowed to dry then sterile forceps placed on MHA antibiotic disks with different potency. Resistance to methicillin was observed using cefoxitin $30 \mu \mathrm{g}$ disks while conducting antibiotic susceptibility tests (Hi-Media Labs, India), and a size inhibition zone of $<21 \mathrm{~mm}$ was considered immune. In vitro antibiotic susceptibility test was done, various antibiotic discs used were penicillin (10 units), ciprofloxacin $(5 \mu \mathrm{g})$, clindamycin $(2 \mu \mathrm{g})$, erythromycin $(15 \mu \mathrm{g})$, levofloxacin $(5 \mu \mathrm{g})$, linezolid $(30 \mu \mathrm{g})$, rifampin $(5 \mu \mathrm{g})$, tetracycline $(30 \mu \mathrm{g})$, and co-trimoxazole $(1.25$ / $23.75 \mu \mathrm{g}$ ). The reference strain used for standardizing antibiotics susceptibility determination was $S$. aureus ATCC 29213. Diameters of the zone of inhibition were measured, and interpretation for determining sensitive, intermediate, or resistant was done according to standard CLSI guidelines". ${ }^{11}$

Determination of Mupirocin-Resistant (MupR) CoNS "Mupirocin resistance among CoNS was assessed using $5 \mu \mathrm{g}$ and $200 \mu \mathrm{g}$ mupirocin discs by Kirby-Bauer disc diffusion method. Isolates with zone $>14 \mathrm{~mm}$ for $5 \mu \mathrm{g}$ as well as $200 \mu \mathrm{g}$ discs was considered as sensitive, isolates with zone $<14 \mathrm{~mm}$ for $5 \mu \mathrm{g}$ but more than $14 \mathrm{~mm}$ for $200 \mu \mathrm{g}$ disc were considered as MupRL, and isolates with zone less than $14 \mathrm{~mm}$ for both 5 $\mu \mathrm{g}$ and $200 \mu \mathrm{g}$ discs was considered as MupRH".12

\section{Epsilometer (E) Test for Determination of Minimum Inhibitory Concentration}

"Kirby Bauer's disc-diffusion technique performed an e-test with HiComb mupirocin strip according to CLSI guidelines. Lawn cultivation was prepared on a medium surface of MHA. HiMedia E strip with mupirocin antibiotic ranged from 0.064 $1240 \mu \mathrm{g} / \mathrm{mL}$ by softly pressing sterile forceps to perfection. The slabs were then aerobically incubated for 24 hours at $35^{\circ} \mathrm{C}$. Checking for the minimum inhibitory concentration (MIC) after incubation plates. MIC isolates $>512 \mu \mathrm{g} / \mathrm{mL}$ were treated as MupRH, MICs $8-256 \mu \mathrm{g} / \mathrm{mL}$ were treated as MupRL and < $4 \mu \mathrm{g} / \mathrm{mL}$ were treated as mupirocin responsive". 13 


\section{Statistical Analysis}

The collected data was tabulated in an Excel Sheet. Data was analysed using IBM SPSS Statistics Windows, version 20.0 IBM Corp., (Armonk, NY, USA) for the generation of descriptive and inferential statistics.

\section{RESULTS}

Overall 100 CoNS isolates were obtained. Antibiotic susceptibility test of these isolates using cefoxitin $30 \mu \mathrm{g}$ disc showed that $24(24 \%)$ isolates were methicillin-resistant CoNS (MRCoNS) and 76 (76\%) isolates were methicillinsensitive CoNS (MSCoNS).

\begin{tabular}{|c|c|c|c|c|c|c|}
\hline \multirow[t]{2}{*}{ Isolate (100) } & \multicolumn{2}{|c|}{$\begin{array}{c}\text { Mupirocin } \\
\text { Sensitive } \\
\text { (MupS) }\end{array}$} & \multicolumn{2}{|c|}{$\begin{array}{c}\text { Low-Level } \\
\text { Mupirocin } \\
\text { Resistance } \\
\text { (MupRL) }\end{array}$} & \multicolumn{2}{|c|}{$\begin{array}{c}\text { High-Level } \\
\text { Mupirocin } \\
\text { Resistance } \\
\text { (MupRH) }\end{array}$} \\
\hline & $\mathrm{N}$ & $\%$ & $\mathrm{~N}$ & $\%$ & $\mathrm{~N}$ & $\%$ \\
\hline $\begin{array}{c}\text { MRCoNS (24) (24 } \\
\%)\end{array}$ & 17 & 70.8 & 4 & 16.6 & 3 & 12.5 \\
\hline $\begin{array}{c}\text { MSCoNS (76) (76 } \\
\%)\end{array}$ & 71 & 93.4 & 3 & 4.2 & 2 & 66.6 \\
\hline
\end{tabular}

\begin{tabular}{|ccc|}
\hline E-test MIC Range $(\boldsymbol{\mu g} / \mathbf{~ m l})$ & \multicolumn{2}{c|}{ No. of Isolates (100) } \\
\hline Sensitive $(<4)$ & N & $\%$ \\
Low-Level Resistance $(8-256)$ & 88 & 88 \\
High-Level Resistance $(>512)$ & 7 & 7 \\
\hline Table 2. MIC of Mupirocin against CoNS & 5 \\
\hline \multicolumn{2}{|c|}{} \\
\hline
\end{tabular}

Overall occurrence of mupirocin resistance was $12 \%$ among all CoNS isolates. Mupirocin resistance was found $7 \%$ in MRCoNS and $5 \%$ in MSCoNS. MupRH and MupRL were $7 \%$ and $5 \%$ respectively (Table 1 ). Out of 100 isolates, majority isolates (88\%) showed less than $4 \mu \mathrm{g} / \mathrm{mL}$ MIC. 7 (7\%) isolates were found to have MIC between $8-256 \mu \mathrm{g} / \mathrm{mL}$ followed by 5 ( $5 \%$ ) isolates were showed MIC more than 512 $\mu \mathrm{g} / \mathrm{mL}$ as described in Table 2 .

\begin{tabular}{|ccccc|}
\hline Antibiotic & \multicolumn{2}{c}{ MSCoNS, N = 76 (\%) } & \multicolumn{2}{c|}{ MRCoNS, N = 24 (\%) } \\
N & $\mathbf{N}$ & $\mathbf{\%}$ & $\mathbf{N}$ & $\mathbf{\%}$ \\
Penicillin & 23 & 30.26 & 24 & 100 \\
Erythromycin & 13 & 17.11 & 13 & 54.17 \\
Clindamycin & 15 & 19.74 & 17 & 70.83 \\
Cefoxitin & 0 & 0.00 & 24 & 100.00 \\
Ciprofloxacin & 6 & 7.89 & 7 & 29.17 \\
Levofloxacin & 3 & 3.95 & 3 & 12.50 \\
Linezolid & 0 & 0.00 & 0 & 0.00 \\
Rifampin & 3 & 3.95 & 21 & 87.50 \\
Co-Trimoxazole & 22 & 28.95 & 15 & 62.50 \\
Tetracycline & 15 & 19.74 & 11 & 45.83 \\
\hline Table 3. Antibiotic Resistance Pattern of Methicillin-Sensitive \\
Coagulates Negative Staphylococcus and Methicillin-Resistant \\
\multicolumn{4}{r}{ Coagulates Negative Staphylococcus Isolates } \\
\hline \multicolumn{5}{c}{} \\
\hline
\end{tabular}

The strains which were significantly coexisting with major antibiotic resistance were MRCoNS strains with $100 \%$ resistance to penicillin, and a higher resistance to rifampin (87.50\%), clindamycin (70.83\%), co-trimoxazole (62.50\%), and erythromycin (54.17\%). All CoNS isolates were equally susceptible to linezolid (Table 3 ).

\section{DISCUSSION}

Advancement in medical sciences with development and the use of medical implants and devices has prolonged the human life span on one hand, although it led CoNS, commensal bacteria, on the other hand, to become a source of hospitalacquired infections. A significant etiological agent of severe life-threatening blood stream infections, the central nervous system shunts prosthetic joints, and preterm babies. S. Aureus and CoNS are significant causes of nosocomial and community-acquired infections. ${ }^{14}$ They are more frequently present in clinical microbiology laboratories as isolated bacterial flora. ${ }^{15}$ CoNS has an extraordinary capability to gather elements of antibiotic resistance, such as penicillin, clindamycin, macrolide, tetracycline, and methicillin, and lately, mupirocin (MUP), and vancomycin. It results in the development of multidrug-resistant strains which reduce the treatment options for infections caused by these species.

"Mupirocin is used as a topical antibacterial that demonstrates a higher degree of streptococci and staphylococci evading infection, and few gram-negative bacteria, including Haemophilus influenza and Neisseria gonorrhoeae, but is much less active against most gramnegative bacilli and anaerobes. It is also used in the treatment of skin infection and for the prevention of MRSA. Mupirocin was used in clinical practice in 1985 and is slowly expanded globally in the form of ointment, after its application. Mupirocin-resistant clinical isolates were first recorded in 1987 with upsurge in the rate of resistance". ${ }^{16}$ Extended usage and regular usage of mupirocin is thought to be a significant factors correlated with mupirocin resistance rise. ${ }^{16}$ Simple and fast defining and discriminating against $S$. aureus, in order to administer effective antimicrobial therapy promptly and to minimize the unnecessary use of a few groups of antibiotics, aureus and CoNS, MET resistance and MUP resistance identification are important. Mupirocin is a topical antibiotic originally isolated from Pseudomonas fluorescens and by competitively binding to isoleucyl-tRNA synthetase (IleS), it inhibits bacterial protein synthesis. This drug is especially effective against streptococci and staphylococci like Staphylococcus aureus (MRSA) which is immune to methicillin. Mupirocin is one of the most common topical agents used to treat skin infection and MRSA nasal carry eradication. Increased use of this antibiotic has been followed by mupirocin-resistant MRSA outbreaks.

In the present study, mupirocin resistance was found among $12 \%$ of CoNS isolates i.e. $7 \%$ in MRSA and $5 \%$ in MSSA. MupRH and MupRL were $7 \%$ and $5 \%$ respectively (7\%). Isolates were found to have MIC between $8-256 \mu \mathrm{g} / \mathrm{mL}$ followed by 5 ( $5 \%$ ) isolates showed MIC more than $512 \mu \mathrm{g} /$ $\mathrm{mL}$ in the present study. These findings were identical to the investigations of Agarwal L et al, ${ }^{17}$ i.e. methicillin resistance was found among $7.6 \%$ CoNS isolates which are higher as reported by Akhtar $(2.1 \%)^{18}$ and much lower than 18 - $60 \%$ reported elsewhere. ${ }^{19-20}$ Differences in the prevalence of CoNS, MRCoNS, and MupRCoNS might be due to the discrepancy in the methodology of recognition used in the laboratories, knowledge, practices, and policies of the institution.

CoNS have a potential tendency to cumulate antibiotic resistance determinants such as to penicillin, clindamycin, macrolide, tetracycline, and methicillin and, in recent times, mupirocin (MUP), and vancomycin. This embarked to the formation of multidrug-resistant strains which reduce the options for the therapy of diseases resulting due to these organisms. Authors of different studies across the globe have used different antibiotics to establish the pattern of antibiotic susceptibility of CoNS and found that MRCoNS were 
multidrug-resistant and recommend performing antibiotic sensitivity test before treatment of infections caused by these species. MRCoNS is significantly correlated with antibacterial resistance such as penicillin, rifampin, clindamycin, Trimethoprim/sulphamethoxazole, erythromycin, and ciprofloxacin in our study. Rahman et al, ${ }^{21}$ studied the CoNS clinical isolates and found in decreasing order the percentage of isolates resistant to oxacillin ( $45 \%$ ), followed by gentamicin (40\%), cefuroxime (31\%), ceftriaxone (30\%), ciprofloxacin (22\%), and none resistant to imipenem and vancomycin and Shah et al,22 found $>50 \%$ of the clinical CoNS isolates were resistant to cephalosporins, aminoglycosides, quinolones, and no organisms resistant to vancomycin. Mupirocin resistance, especially high-level resistance, provides less treatment options for topical use. Another cause for concern is the existence of comparatively higher levels of mupirocin resistance in CoNS. Studies say that mupA gene known to be encoding for resistance to mupirocin could be transferred from MRSA S. epidermidis during prophylaxis of mupirocin, which could pose a significant threat to future use of mupirocin against MRSA.

Higher carriage rate for CoNS, MRCoNS, and MupRCoNS seen in rural population may be partially related to their personal hygiene. Increased use of mupirocin in hospital leads to surge in high-level resistance to mupirocin \& resistance to other CoNS antibiotics. It might impact the treatment of prosthetic infections leading to increase in risk of high-level mupirocin resistance among $S$. aureus over the long term.

\section{CONCLUSIONS}

Enhanced mupirocin usage is correlated with a faster surge in higher level mupirocin resistance and also resistance to various other antibacterials in CoNS. This might exhibit tangible consequence in treating infections and might, in the long run, enhance the risk of $S$. aureus for greater level of resistance to mupirocin. In case of development of mupirocin resistance, other decolonization options should be considered.

Financial or other competing interests: None.

\section{REFERENCES}

[1] Isaacs D. A ten year, multicentre study of coagulase negative staphylococcal infections in Australasian neonatal units. Arch Dis Child Fetal Neonatal Ed 2003;88(2):F89-93.

[2] van den Hoogen A, Gerards LJ, Verboon-Maciolek MA, et al. Long-term trends in the epidemiology of neonatal sepsis and antibiotic susceptibility of causative agents. Neonatology 2010;97(1):22-8.

[3] Patil R, Patil T, Hussain KMA. Staphylococcus lugdunensis native tricuspid valve endocarditis: a case report and review of literature. J Gen Intern Med 2011;26(10):120911.

[4] Shah MU, Akram MF, Usman J, et al. Incidence and susceptibility pattern of methicillin resistant coagulasenegative staphylococci isolated from a tertiary care hospital of Pakistan. Jundishapur J Microbiol 2014;7(1):e8590.

[5] Chu VH, Woods CW, Miro JM, et al. Emergence of coagulase-negative staphylococci as a cause of native valve endocarditis. Clin Infect Dis 2008;46(2):232-42.

[6] Zhang K, Sparling J, Chow BL, et al. New quadriplex PCR assay for detection of methicillin and mupirocin resistance and simultaneous discrimination of Staphylococcus aureus from coagulase-negative staphylococci. J Clin Microbiol 2004;42(11):4947-55.

[7] Diekema DJ, Pfaller MA, Schmitz FJ, et al. Survey of infections due to Staphylococcus species: frequency of occurrence and antimicrobial susceptibility of isolates collected in the United States, Canada, Latin America, Europe, and the Western Pacific region for the SENTRY Antimicrobial Surveillance Program, 1997-1999. Clin Infect Dis 2001;32 Suppl 2:S114-32.

[8] Park SY, Kim SM, Park SD. The prevalence, genotype and antimicrobial susceptibility of high- and low-level mupirocin resistant methicillin-resistant Staphylococcus aureus. Ann Dermatol 2012;24(1):32-8.

[9] Anthony RM, Connor AM, Power EG, et al. Use of the polymerase chain reaction for rapid detection of highlevel mupirocin resistance in staphylococci. Eur J Clin Microbiol Infect Dis 1999;18(1):30-4.

[10] Mehrishi P, Faujdar SS, Kumar S, et al. Antibiotic susceptibility profile of uropathogens in rural population of Himachal Pradesh, India: Where we are heading? Biomed Biotechnol Res J 2019;3(3):171-5.

[11] Clinical and Laboratory Standards Institute. Performance standards for antimicrobial susceptibility testing; twentysecond informational supplement. Vol. 34. Wayne, Pennsylvania, USA: Clinical Laboratory Standard Institute 2014:68-75.

[12] Clinical and Laboratory Standard Institute. Performance standards for antimicrobial susceptibility testing. Wayne, PA, USA: Clinical and Laboratory Standard Institute 2010.

[13] Chaturvedi P, Singh AK, Singh AK, et al. Prevalence of mupirocin resistant staphylococcus aureus isolates among patients admitted to a tertiary care hospital. $\mathrm{N} \mathrm{Am}$ J Med Sci 2014;6(8):403-7.

[14] Hussain Z, Stoakes L, Massey V, et al. Correlation of oxacillin MIC with mecA gene carriage in coagulasenegative staphylococci. J Clin Microbiol 2000;38(2):7524.

[15] von Eiff C, Peters G, Heilmann C. Pathogenesis of infections due to coagulase-negative staphylococci. Lancet Infect Dis 2002;2(11):677-85.

[16] Patrick CC. Coagulase-negative staphylococci: pathogens with increasing clinical significance. J Pediatr 1990;116(4):497-507.

[17] Agarwal L, Singh AK, Agarwal A, et al. Methicillin and mupirocin resistance in nasal colonizers coagulase negative Staphylococcus among health care workers. Med J DY Patil Univ 2016;9(4):479-83.

[18] Akhtar N. Staphylococcal nasal carriage of health care workers. J Coll Physicians Surg Pak 2010;20(7):439-43.

[19] Rao KA, Deepa S, Venkatesha D. Screening for nasal colonizers: mandatory to prevent surgical site infections. Int J Sci Study 2014;2:1-5. 
[20] Lee YL, Cesario T, Tran C, et al. Nasal colonization by methicillin-resistant coagulase-negative Staphylococcus in community skilled nursing facility patients. Am J Infect Control 2000;28(3):269-72.

[21] Rahman A, Hossain MA, Paul SK, et al. Methicillinresistant coagulase-negative staphylococci (MRCoNS) by disk diffusion method. Mymensingh Med J 2013;22(2):229-31.
[22] Shah MU, Akram MF, Usman J, et al. Incidence and susceptibility pattern of methicillin resistant coagulasenegative staphylococci isolated from a tertiary care hospital of Pakistan. Jundishapur J Microbiol 2014;7(1):e8590. 